\title{
The Biology of Nicotine Dependence (Ciba Foundation Symposium 152)
}

E. F. Domino

Dept Pharmacology, University of

Michigan, Ann Arbor, Ml 48109-0626,

USA. edited by Greg Bock and Joan Marsh, John Wiley \& Sons, 1990. E35.95 (ix +264 pages) ISBN 0471926884

The organizers of this Ciba Foundation Symposium brought together an outstanding group of scientists whose research has contributed much to our understanding of the complex actions of nicotine. This volume summarizes the papers and discussions presented at that symposium held 7-9 November, 1989.

The emphasis of this small book is on the pharmacology of nicotine with only limited information on its liability to induce dependence. Especially interesting are the discussion sections after each chapter. These are most helpful and give the reader a sense of what it was like at the symposium. The final discussion on the withdrawal syndrome induced by nicotine and on possible therapeutic approaches, including the use of transdermal nicotine patches as an aid to people trying to give up their smoking habit, is quite valuable. The general dis- cussion sections dealing with the activation, location and function of nicotinic cholinergic receptors, and the discussion on adaptive and cognitive aspects of nicotine are noteworthy.

A wide variety of subjects has been covered in this volume including molecular receptor relationships, the pharmacological actions of nicotine via nicotinic cholinergic receptors, and the pre- and postsynaptic localization of these receptors. Those subjects that deal with the issue of nicotine receptors are of great interest and the whole book is certainly a significant contribution to our knowledge of this field. For example, there are seven genes that code for $\alpha$ and $\beta$ subunits; these all exist in the brain and there are therefore many possible different nicotinic receptors. There are four different $\alpha$ ligandbinding units and three $\beta$ subunits that form a muscle type receptor.

I was somewhat disappointed that there was no detailed discussion of the actions of nicotine through receptor mechanisms other than those that are nicotinic cholinergic in nature. There is evidence in the literature that nicotine has actions on receptors other than those that have strictly nicotinic cholinergic properties. Any discussion on the subject of nicotine dependence should cover why chronic administration causes up-regulation of nicotinic receptors in the brain rather than down-regulation (as is true of almost all other pharmacological agents given chronically). This discovery should be explored in great detail and might have considerable significance with respect to the subject of nicotine dependence. This crucial aspect of nicotine pharmacology on chronic administration was not adequately covered. Endocrine, metabolic, CNS, behavioral reinforcing properties, and the pharmacokinetic and pharmacodynamic aspects of nicotine were covered very well. Obviously, much has been learned in the past hundred years since J. N. Langley and associates carried out their classical studies of nicotine and first gave a valid description of the concept of receptors.

In spite of our extensive knowledge of nicotine, it is surprising that relatively little is known about nicotine dependence and withdrawal. This is not the fault of the symposium organizers and participants but rather indicates the need for much further study.

\section{Analysis of Neuronal Microcircuits and Synaptic Interactions (Handbook of Chemical Neuroanatomy, Vol. 8)}

John Parnavelas

Dept Anatomy and Developmental Biology, University College London,

Gower Street, London WC1E 6BT, UK. 527 pages) ISBN 0444812318 edited by A. Björklund, T. Hökfelt, F. G. Wouterlood and A. N. van den Pol, Elsevier, 1990. Dfl. 400.00 ( $x x+$

This, together with the previous seven volumes of this series, contains invaluable information for those interested in the localization and function of putative transmitter substances in the nervous system. The editors are some of the pioneers of the rapidly expanding field of chemical neuroanatomy. This field was first established in the early 1960 s with the development of the catecholamine histofluorescence methods by Falck and Hillarp and of the acetylcholinesterase staining a few years later by Shute and Lewis.

The first volume of the series appeared in 1983 and it was dedicated to a comprehensive coverage of the major techniques used in chemical neuroanatomy at that time. However, many technical developments have occurred during the past seven years. Along with improved tract-tracing and immunocytochemical methods, innovative combinations of techniques have been developed that provide extremely powerful tools for extracting maximum information either from individual neurones or from assemblies of cells. The present volume complements and updates Volume 1 by focusing on recent developments and particularly on approaches that combine neuroanatomical, histo- chemical, immunohistochemical and neurophysiological techniques. The chapters included in this volume are scholarly reviews, complete with theoretical backgrounds of the methods, discussions of their advantages and limitations as well as detailed recipes. After all, readers of this volume will be primarily those interested in applying a particular technique, and I am certain they will appreciate the practical detail given in most chapters - vital information that is often missing from the Methods sections of research papers. For example, the chapter by Groenewegen and Wouterlood about the use of the lectin PHA-L includes details such as the source and batch number of PHA-L and the source and types of antisera - information that was undoubtedly missing from the protocols used by 